\title{
OBSERVATION OF VISIBLE SYNCHROTRON RADIATION EMITTED BY A HIGH-ENERGY PROTON BEAM AT THE EDGE OF A MAGNETIC FIELD
}

\author{
R. BOSSART. J. BOSSFR, L. BI RNOE, R. COISSON* IE I) AMICO. A. HOEMANN and J. MANN

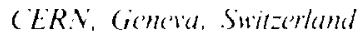

Recuived 22 March 1979

\begin{abstract}
Theoretical studies show that owing to the abrupt shange of the magnetic tield occurring at the magned edges synehrouron radiation will be emitted in the sisible light range, by a high-energy proton beam.

Experiments have been carried out at the (I:RN Super Proton Synchrotron (SPS) in order to check for the validity of the theory and measure the properties of the emilted light.

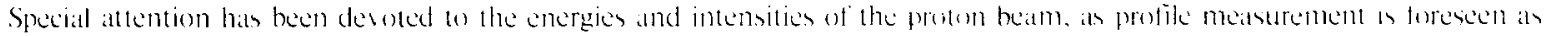
an immediate applicatlom.
\end{abstract}

\section{Introduction}

Synchrotron radiation is a well-known effect as far as ultrarelativistic electrons are concerned. For protons with energies up to $400 \mathrm{GeV}$ the classical theory shows that only a negligible part of the synchrotron radiation power is in the range of the visible light spectrum.

However an earlier paper') showed that, in the case of a high-energy proton synchrotron the magnetic field discontinuity at the edges of the bending magnets will shift the spectrum to the visible region where the usual light detectors can be used.

Calculations were carried out ${ }^{\prime}$ ) assuming a Gaussian transition for the edge field of the magnets. The results obtained were promising. In order to check the validity of the theory, an experiment was carried out at the CERN SPS $400 \mathrm{GeV}$ accelerator.

\section{Physical principles}

Symbols used:

$m$ : proton mass,

$e \quad$ : proton charge.

$B \quad$ : magnetic field

$R \quad$ : radius of curvature.

C : speed of light.

$E \quad$ : particle energy.

$E_{01} \quad:$ rest energy $=0.938 \mathrm{GeV}, \gamma=E / E_{01}$.

" : particle speed, $\beta=1 / / c$,

$\theta \quad$ : angle of observation.

$l$ : frequency,

$n \quad:$ number of protons per second,

$\mathrm{d} P / \mathrm{d} \Omega$ : power per unit solid angle,

$I \quad$ : proton beam intensity

$p \quad$ : number of protons circulating in the accelerator.

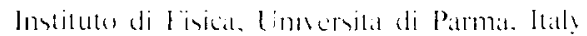

$r \quad:$ unit vector from proton to observer.

An ultrarelativistic $(\xi \geqslant 1)$ charged particle travelling in a transversal uniform magnetic field $B$ emits synchrotron radiation, mostly confined in a cone of aperture $1 / \%$ its axis being the tangent to the particle trajectory ${ }^{2.3}$ ).

An observer will detect this electromagnetic field only when the tangent to the particle trajectory makes an angle $\leqslant 1 / \gamma$ on either side of the straight line joining the particle to the observation point (fig. 1).

It is easy to prove that ${ }^{\prime}$ )

$L_{1}=\frac{R}{\because}=\frac{m c}{i B}$

is the length over which the electromagnetic (e.m.) field is produced. The corresponding observation time is

$\tau_{0}=\tau_{1}(1-\beta)=\frac{\tau_{11}}{2 \gamma^{2}}=\frac{R}{20 \eta^{3}}$.

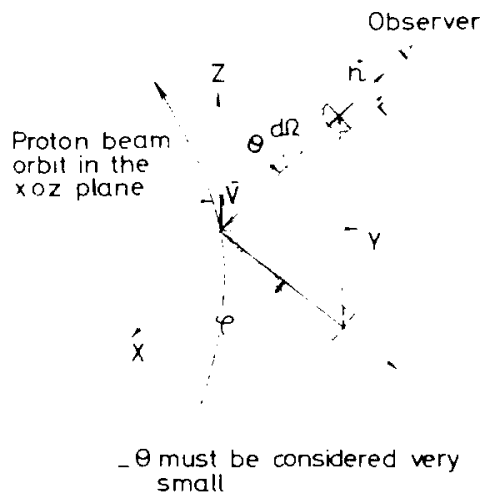

I"ig. 1. Simbols usicl 
Thus the observer detects a pulse of width $\tau_{\mathrm{c}}$ at each passage of the particle. The spectral analysis shows non-negligible components for frequencies at least up to $v_{\mathrm{c}}=1 / \tau_{\mathrm{c}}$. The spectral density decreases as $\left(v / v_{\mathrm{c}}\right)^{1 / 2} \exp \left(-4 \pi v / 3 v_{\mathrm{c}}\right)$ when $v>v_{\mathrm{c}}$. The quantity $3 v_{\mathrm{c}} / 4 \pi$ is often called "critical frequency".

If a rapid variation from $B=B_{0}$ to $B=0$ (or conversely) occurs within a distance $L<L_{0}$, the previous spectrum is extended. More precisely the critical frequency is shifted to a higher value. If the observer is looking at the edge along the projection of the straight section of the trajectory, the fall- or rise-time is about ${ }^{1}$ )

$\tau_{\mathrm{d}} \simeq \frac{L}{c} \frac{1}{2 \gamma^{2}}$.

In the SPS, where $R=740 \mathrm{~m}, L=0.1 \mathrm{~m}$ and for $E=270 \mathrm{GeV}, \lambda_{\mathrm{c}}=c \tau_{\mathrm{c}}=16 \mu \mathrm{m}, \lambda_{\mathrm{d}}=c \tau_{\mathrm{d}}=0.6 \mu \mathrm{m}$. $\lambda_{\mathrm{d}}$ is then in the visible red while $\lambda_{\mathrm{c}}$ stays in the far infrared.

The number of photons emitted in the visible spectrum is calculated as follows.

We can consider the spectrum extended to the frequency $\nu_{\mathrm{d}}=1 / \tau_{\mathrm{d}} \gg 1 / \tau_{\mathrm{c}}$. If we approximate $\tau_{\mathrm{c}}$ as infinitely long, the only contribution comes from the edge which produces a deflection $\ll 1 / \gamma$. The problem is then similar to that of a "short magnet".

Considering the usual formulae ${ }^{3}$ ) derived from the Lienard-Wiechert potential, and taking into account that $\gamma \gg 1, \theta \ll 1$, that is

$(1-\beta \cos \theta) \simeq\left(1+\theta^{2} \gamma^{2}\right) / 2 \gamma^{2}$, the e.m. field amplitude is (incoherent addition of amplitudes of each proton):

$U(t)=K I^{2} \gamma^{3} B f(0, \phi)$,

where $B$ and $f$ must be evaluated at time

$t^{\prime}=t-\frac{r(t)}{c} \simeq \frac{2 \gamma^{2} t}{1+\gamma^{2} \theta^{2}}$,

$K^{2}=\frac{e^{3}}{\pi^{2} \varepsilon_{0} m^{2} c}=0.056$ MKSA units,

and

$f(0, \phi)=\left(1+\gamma^{2} \theta^{2}\right)^{-3}\left[\left(1+\gamma^{2} \theta^{2}\right)^{2}-4 \gamma^{2} \theta^{2} \cos ^{2} \phi\right]^{\frac{1}{2}}$,

(see fig. 1).

The power spectrum $\mathrm{d} P / \mathrm{d} \Omega \mathrm{d} \nu$ is then given by $\frac{\partial P}{\partial \Omega C^{2} v}=K^{2} I y^{6} f^{2} \cdot \mathscr{F}^{2}[(B(t)]$,

where $\bar{F}^{2}[B(t)]$ is the modulus square of the $B(t)$
Fourier transformed. The integrated spectrum $\mathrm{d} P$ / $\mathrm{d} v$ (collected within an angle $\theta \simeq 1 / \gamma$ ) is

$\frac{\mathrm{d} P}{\mathrm{~d} v}=\pi K^{2} I \gamma^{4} \int_{1}^{\infty} \overline{[f(y)]^{2}} \mathscr{\mathscr { H }}^{2}[B(t)] \mathrm{d} y$

where $\left[\overline{f(y)}^{2}\right.$ is the average of $f$ over $\phi$, $y=1+\gamma^{2} \theta^{2}$ and therefore $\mathrm{d} y=2 \gamma^{2} \theta \mathrm{d} \theta$, the integration limits being 1 and $\infty$.

It is more convenient [to avoid making many Fourier transforms (FT) with different time scales as $t$ depends on the angle] to make the FT of $B$ as a function of $t^{\prime}$, which is independent of the angle, and to perform a change of variable. Of course the frequency scale has to be expanded by a factor $2 \gamma^{2} / y$

$$
\begin{aligned}
\frac{\mathrm{d} P}{\mathrm{~d} v} & =\frac{\pi}{4} K^{2} I \int_{1}^{\infty} y^{2} \overline{[f(y)]^{2}} \widetilde{B}^{2}\left(\frac{y^{\nu}}{2 y^{2}}\right) \mathrm{d} y \\
& =\frac{\pi}{4} K^{2} I \int_{1}^{\infty}\left(y^{-2}-2 y^{-3}+2 y^{-4}\right) \tilde{B}^{2}\left(\frac{y v}{2 \gamma^{2}}\right) \mathrm{d} y,
\end{aligned}
$$

where $\widetilde{B}\left(v^{\prime}\right)=\widetilde{F}\left[B\left(t^{\prime}\right)\right]$.

The theoretical case of an error function for $B(z)$ has been analysed elsewhere ${ }^{4}$ ). In our case these calculations have been carried out with a computer, taking into account the measured magnetic end fields of the SPS magnets. Their results will be described in section 5 .

The emitted light is polarized. Two electrical field components $E_{\mathrm{r}}$ and $E_{\pi}$ have to be considered as shown in fig. 2. The radiated intensity in plane $E_{r}$ is an order of magnitude less than the corresponding value for $E_{\mathrm{r}}$ and depends on which part of the spectrum the observation is made. $E_{\pi} / E_{\mathrm{r}}=1 / 7$ for the whole spectrum and tends to go to 0 if only the highest frequencies are selected.

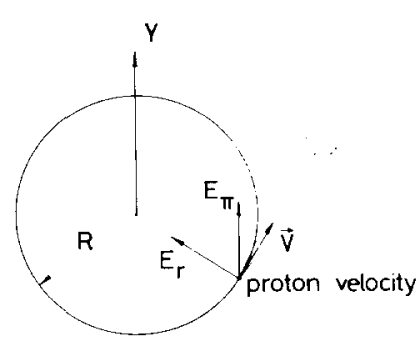

$-E_{\mathrm{r}}$ : on the proton orbit plane

$-E_{\pi}$ : perpendicular to $E_{\mathrm{r}}$

Fig. 2. Polarization of the light 


\section{Experimental set-up}

In the SPS, the guiding field is provided by 746 identical bending magnets, each producing a deflection of $8.4 \mathrm{mrad}$. The synchrotron light, emitted by the trailing edge of one magnet $\mathrm{Bl}$ and the leading edge of the following magnet $\mathrm{B} 2$ at a distance of $40 \mathrm{~cm}$ from each other (fig. 3), will reach the required clearance from the proton beam after a given distance and later on will hit the vacuum chamber.

A flat surface mirror, installed inside the vacuum chamber at the optimum distance from the emission region $(11.6 \mathrm{~m})$, reflects the synchrotron light through a glass window without disturbing the circulating beam.

With the existing vacuum chamber, the mirror cannot be made large enough to intercept all the radiated light contained in the cone.

The receiver looking at the light which escapes from the window was either a vidicon followed by a TV set or a photomultiplier.

The size of the light source is given by the proton beam sizes. At high energy ( $\geqslant 250 \mathrm{GeV})$ and high intensity $\left(\geqslant 10^{13} \mathrm{ppp}\right.$ ), where the radiation is observable, the beam sizes are roughly $3 \times 3 \mathrm{~mm}^{2}$.

Three experiments have been carried out:

1) The first consisted in using a standard TV camera equipped with an RCA silicon target vidicon type 4532 . The vidicon target has a minimum sensitivity of $2 \times 10^{8}$ photons/ $\mathrm{s} / \mathrm{mm}^{2}$. The bandwidth is centred at $635 \mathrm{~nm}$. One could thus observe a spot which brightens during the acceleration time.

A

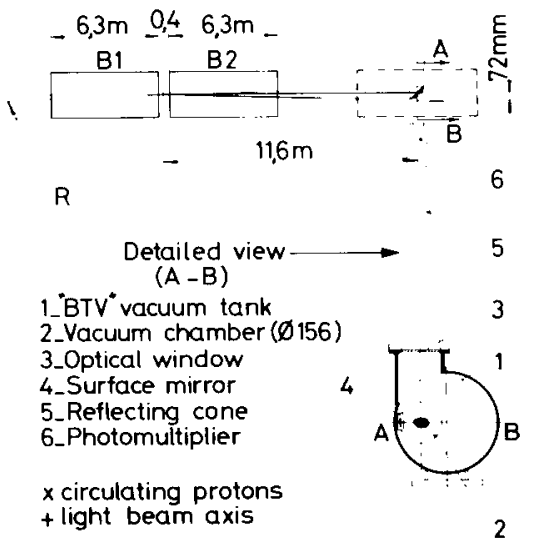

Fig. 3. Experimental set-up.

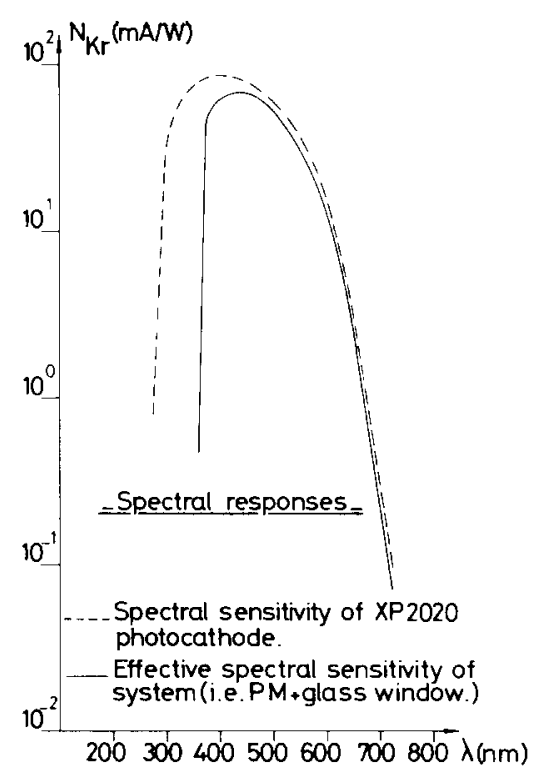

Fig. 4. Spectral responses

2) The second experiment used a photomultiplier (PM) instead of the vidicon. It was placed at the top of a cone in order to be less sensitive to radioactivity. The spectral response of the tube $^{*}$ covers the range from 300 to $500 \mathrm{~nm}$. The gain may reach $10^{8}$. The quantum efficiency is about $20 \%$.

3) The third experiment used the PM together with a plastic type polarizer placed on the glass window of the vacuum chamber. Adequate rotation of the polarization plate allowed us to check for the polarization of the light. Of course reflections on the cone might induce some errors which are negligible in so far as we looked for qualitative results.

Fig. 4 shows the PM spectral response and the resulting PM and window response.

\section{Experimental results}

\subsection{Observation by TV camera}

Nothing but qualitative results were expected from this first experiment. A spot appeared on a TV screen for an energy $>350 \mathrm{GeV}$ and a proton current $>6 \times 10^{12} \mathrm{p}$. It was observed that the brightness increased with energy and beam intensity.

\subsection{Observation by the Photomultiplier}

The output signal of the PM has been simply observed on a scope and also passed through a

\footnotetext{
* Type XP2020 manufactured by Radiotechnique.
} 


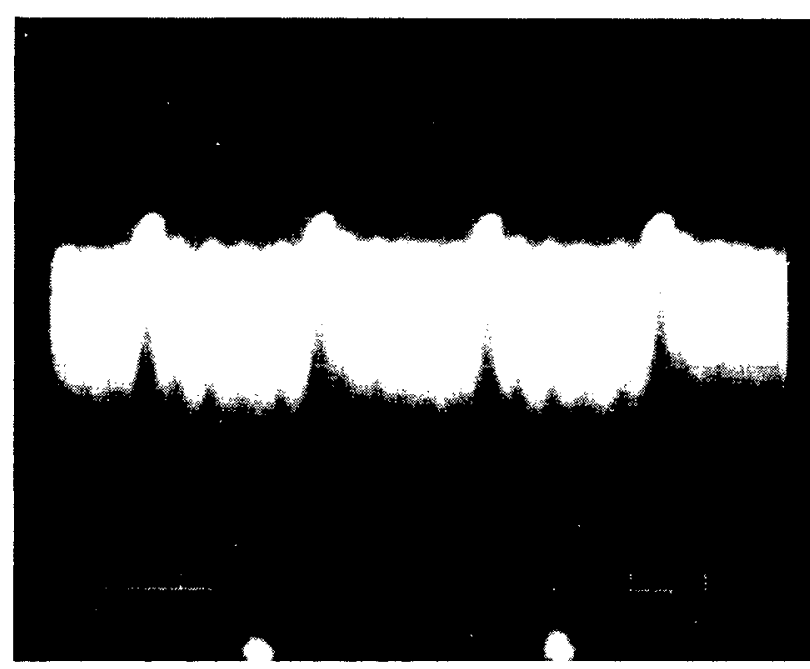

Fig. 5. Direct vutput of the photomultiplier. Scaling: vertical $5 \mathrm{mV} / \mathrm{div}$; horicontal $10 \mu \mathrm{s} / \mathrm{div}$

$100 \mathrm{~Hz}$ low-pass filter before being displayed on a scope.

Fig. 5 shows the direct observation. One can notice an amplitude modulation every $23 \mu \mathrm{s}$, showing that the synchrotron light detected by a PM reflected the time structure of the beam. (23 $\mu$ s is the revolution period).

The filtered output is displayed in figs. $6 a$ and $b$. The signal starts increasing at $270 \mathrm{GeV}$ and rises like an exponential function. The flat top in fig. $6 \mathrm{a}$ is a consequence of a constant energy for the proton beam. Different measurements had been carried out in order to determine roughly the number of generated photons. The gain of the PM, estimated by single photoelectron measurement, was found to be about $8 \times 10^{7}$ at $2600 \mathrm{~V}$. Finally one finds that $4 \times 10^{9}$ photon $/ \mathrm{s}$ correspond to a circulating proton beam of $14 \mathrm{~mA}\left(2 \times 10^{12}\right.$ protons in the ring) at an energy of $400 \mathrm{GeV}$.

Synchrotron light has been detected with the proton beam as weak as $10^{11} \mathrm{p}(0.7 \mathrm{~mA})$.

Linearity is perfect if one refers to the straight decay of fig. $6 \mathrm{~b}$, where immediately after the energy rise a slow uniform extraction of the beam was performed at constant energy.

\subsection{TEST FOR POLARIZATION}

The results of this experiment show that the horizontal polarization is roughly twenty times greater than the vertical polarization.

The quantitative interpretation of the experimental results can hardly be made without the help of computers. As a matter of fact, when the energy
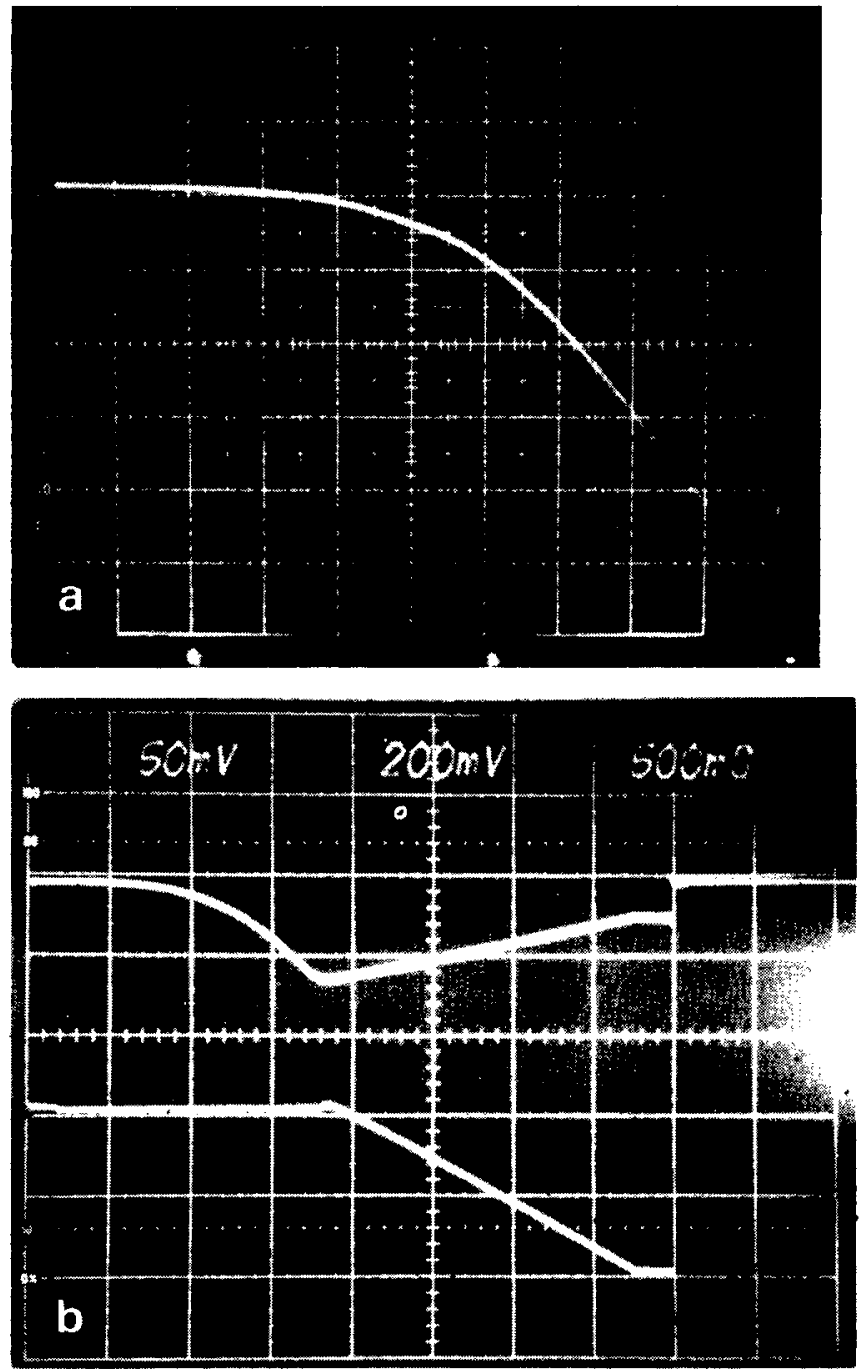

fige. 6. (a) Filterod output of the photomultiplier during energy

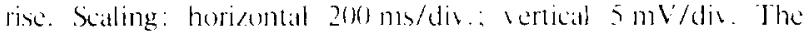
lower end of the trake corresponds to the SPS 400 (id t energy - llat top". (b) Linciarty lest during a blow beam extration at 400) (iel. The upper trace represents the filtered output of the photomultiplier. The lower trace represents the output of the circulating intensity monitor. The slow extratetion starts att about $18010 \mathrm{~ms}$ and ends at $3700 \mathrm{~ms}$ on the picture. At $4(x) \mathrm{m}$. the rematining beam is last cxaracted

rises, the light yield, the spectrum, and the angle of emission change. The next section will deal with this subject.

\section{Analyis of the results}

Using the measured magnetic end fields, the synchrotron radiation spectrum has been computed. The spectrum on the axis for an ideal beam $\partial^{2} P /$ $\partial \Omega \partial v$ is shown in fig. 7. The minima are due to 


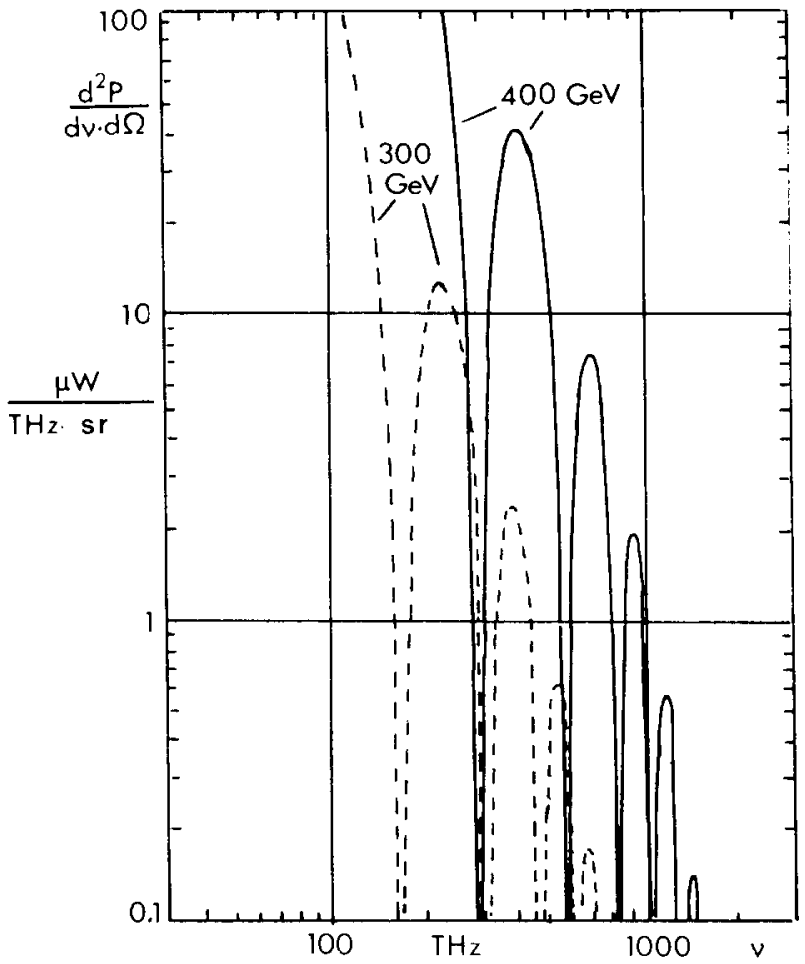

Fig. 7. Computed power spectrum per unit solid angle on the axis $(\theta=0)$ enitted by an ideal proton beam with $I=100 \mathrm{~mA}$ and $E=400 \mathrm{GeV}$ (full line) and $E=300 \mathrm{GeV}$ (dashed line) passing through the magnet discontinuity.

interference of the radiation coming from the two magnet ends. The spectrum is then integrated over all angles, $\theta$ and $\phi$, which are accepted by the experimental set-up. The resulting spectra are shown in fig. 8 for 300 and $400 \mathrm{GeV}$, assuming a beam current of $100 \mathrm{~mA}$. The "ordinary" synchrotron radiation from the long magnets is also shown in fig. 8 for $400 \mathrm{GeV}$; it is negligible in the visible part of the spectrum. In this calculation the angular spread of the protons has not been taken into account; it is actually negligible. The accepted spectrum is integrated over the spectral sensitivity of the photomultiplier. The resulting expected response is shown in fig. 9, together with experimental results. The agreement is quite good, even when taking into account experimental errors.

Analysis of fig. 8 shows that at $300 \mathrm{GeV}$, even at $400 \mathrm{GeV}$, the spectral XP2020 response is not entirely covered. The window transmission factor has not been taken into account, because it affects only the high-frequency spectrum region.

The energy dependence may be approximated, in this energy range, by an expression of the form $g(\gamma)=a \gamma^{8}+b$.

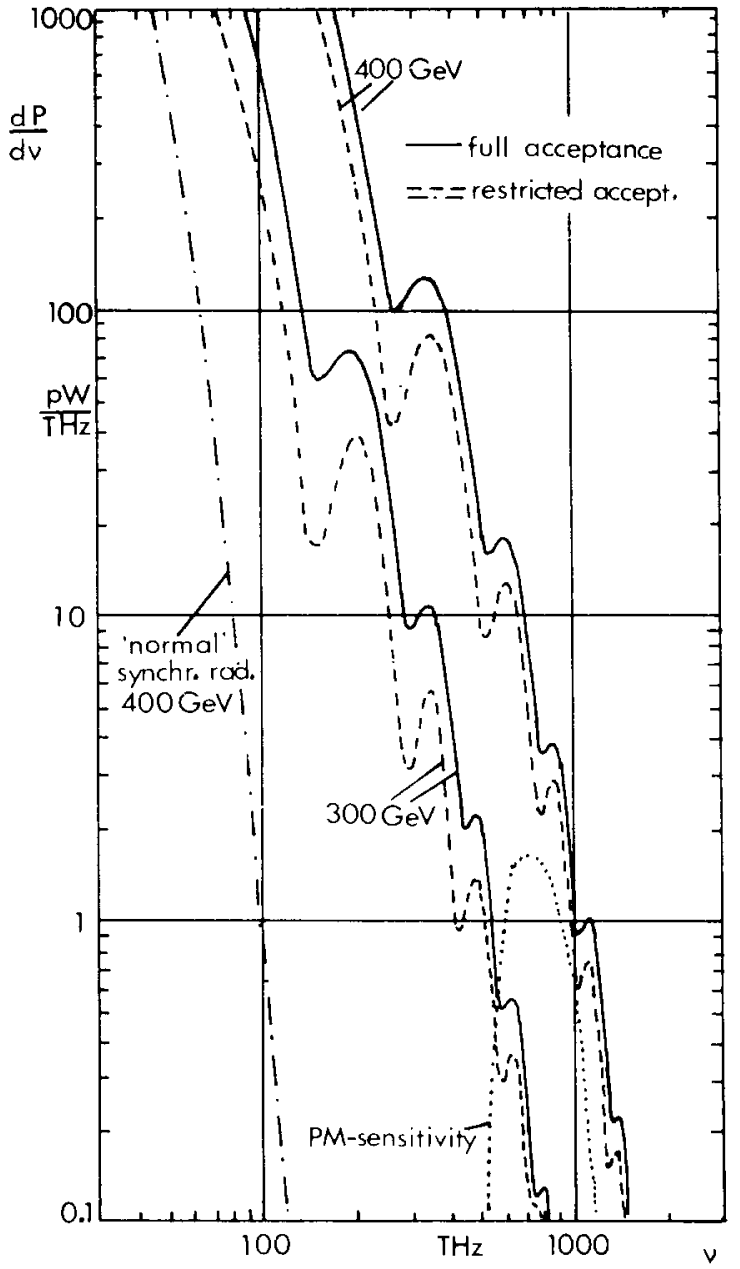

Fig. 8. Computed power spectrum (full line - integrated over all angles: dashed line - integrated over the acceptance of the experimental set-up) emitted by a proton beam (with $I=100 \mathrm{~mA}, E=400$ and $300 \mathrm{GeV}$ ) passing through the magnet discontinuity. For comparison the "normal" synchrotron radiation spectrum enitted at $400 \mathrm{GeV}$ from the long magnets is shown (dash-dotted line). The spectral sensitivity of the photomultiplier (ignoring absorption in the glass window) is plotted (dotted line) in arbitrary units.

\section{Conclusions}

Let us summarize the information obtained by the experiments.

The use of a television camera allowed us to observe a "spot-like" luminous source dependent on the proton beam characteristics. On the other hand, the information given by the photomultiplier was quantitative and provided a good check, according to the theory, for the dependence of the light source on the energy and intensity of the proton beam. Finally, the fact that the measured light was almost horizontally polarized is a comple- 


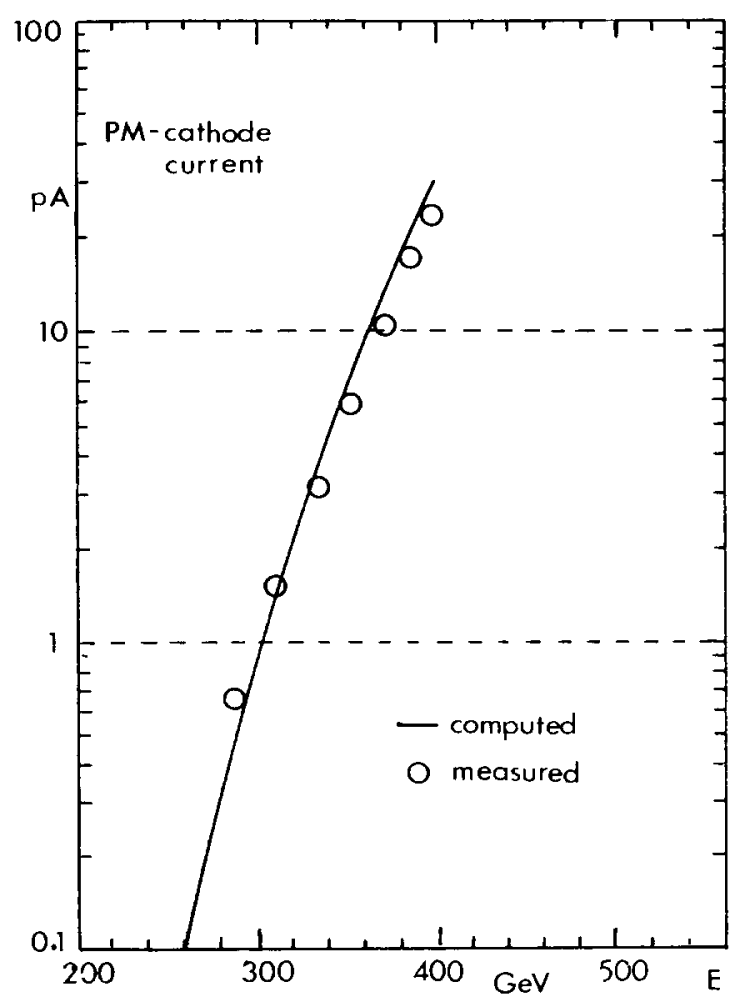

Fig. 9. Computed absolute response (cathode current in pA) of the photomultiplier as a function of proton energy $E$ for $I=100 \mathrm{~mA}$ and comparison with measurements. mentary proof of the synchrotronic origin of the radiation.

The goal of these experiments was to detect for the first time the synchrotron light emitted by a proton beam and to prove the validity of the edge effect.

Furthermore, a direct application will be the beam profile measurements for the future protonantiproton experiments. As the two beams will be circulating in opposite directions, the distinction between $p$ and $\vec{p}$ radiation appears to be easy. A non-intercepting profile measurement of each particle beam for a fixed energy looks feasible at high energy.

This work was strongly supported by B. de Raad. We would like also to thank all the persons who helped us, and in particular J. Camas, A. ChapmanHatchett, J. Donnier, U. Kracht, J. P. Papis and H. Rossi.

\section{References}

1) R. Coïsson, Opt. Commun. 22 (1977) 135

2) R. Coïsson. Nucl. Instr. and Meth. 143 (1977) 241.

3) J. D. Jackson, Classical electrodynamics (J. Wiley, NY, 1962).

4) R. Coïsson, Phys. Rev. A, to be published. 